\title{
Geometric considerations for the design of production-friendly high-speed ship hull forms
}

S W Boyd ${ }^{1 *}$, A H Day ${ }^{2}$, and I E Winkle ${ }^{2}$

${ }^{1}$ Fluid Structure Interactions Research Group, School of Engineering Science, University of Southampton, Southampton, UK

${ }^{2}$ Department of Naval Architecture and Marine Engineering, Universities of Glasgow and Strathclyde, Glasgow, UK

The manuscript was received on 23 July 2004 and was accepted after revision for publication on 7 June 2005.

DOI: $10.1243 / 147509005 X 10486$

\begin{abstract}
This study examines the feasibility of designing high-speed ships with hull-form geometry suitable for planked construction, with the aim of reducing the hull construction cost. An algorithm is developed for placing prismatic planks on to a three-dimensional hull form to represent a planked construction. A number of well-known hull forms are examined using the algorithm developed in order to assess their suitability for this construction technique. It is shown that typical round-bilged forms are unsuitable for planked construction, since an undesirably large proportion of the material strength will be used in forming the structure. A conceptual design for a simplified hull form is developed which contains significantly reduced levels of double curvature, and this design is shown to be suitable for planked construction, as well as offering the potential for advantages in conventional plated construction. It is further shown that the hydrodynamic resistance of this conceptual design is comparable with a more traditional form.
\end{abstract}

Keywords: geometry, design, high-speed ships, hull forms, construction cost

\section{INTRODUCTION}

There is an increasing demand for cost-effective construction of marine vehicles in order to stay competitive in a now world market. Studies have shown that the proportion of ship construction cost attributed to direct labour can be as much as 45 per cent for fast private super-yachts, 33 per cent for commercial fast ferries, and 28 per cent for fast patrol vessels [1]. One method of reducing labour input is to standardize the component parts that make up the ship structure. In recent years, there has been a move towards the use of extruded aluminium components in substantial parts of high-speed ship structures. There are now a variety of standard and proprietary extruded planks, which incorporate stiffeners and edge details to allow rapid and

* Corresponding author: Fluid Structure Interactions Research Group, School of Engineering Science, University of Southampton, Highfield, Southampton SO17 1BJ, UK. email: sbody@ ship.soton.ac.uk economic construction. Similar concepts have been developed utilizing fibre-reinforced polymers (FRPs) in the form of pultruded planks. However, the application of FRP planks has been limited to mainly civil applications $[\mathbf{2}, \mathbf{3}]$.

At present, the use of preformed components (in particular, aluminium extrusions) has been concentrated in areas of the ship in which the geometry largely consists of planar or near-planar surfaces, such as decks, bulkheads, and superstructure panels. However, there are potentially large gains to be made in constructing geometrically more complex structures, such as the hull shell plating, using preformed planks. This is especially true of FRP construction where a plank-based system could reduce, or even eliminate, the need for a female mould.

Traditional ship planking techniques using wood planks employed 'stealers' and tapered planks to help to achieve the required geometry, especially near the ends of the hull where the girth can reduce rapidly. However, the nature of the manufacturing methods for both FRP pultrusions and aluminium extrusions 
limits the plank geometry to purely prismatic sections. While the sections could clearly be cut and tapered, much of the advantage of prefabrication would be lost, especially as tapering the planks would generally mean the loss of the edge detail, and consequent difficulty in joining the planks along seams. Therefore, it is likely that the hull geometry would have to be modified in order to allow the hull to be constructed using only prismatic planks without unacceptably high levels of curvature and associated forming stresses.

Within this study an algorithm has been developed to position planks of a specified constant width on any given three-dimensional surface. From knowledge of the position of each plank on the surface, the curvatures induced on the planks by the surface can be calculated and used to determine the stress induced by forming. There are a number of inputs to the procedure that can have a significant effect on the magnitude of the forming stress including the direction of the planking and/or the start position of the chosen planking strategy.

This paper describes the development of the planking algorithm and the subsequent calculation of curvature and forming stress. The algorithm is applied to a number of hull forms to investigate their possible construction using a planked technique. Design considerations are identified and implemented through the conceptual design of a simplified hull form. This was shown to have similar hydrodynamic resistance to a conventional design of comparable dimensions, while offering substantial advantages in terms of ease of planked construction.

\section{DESCRIPTION OF THE PLANKING ALGORITHM}

\subsection{Surface data}

There are a number of software packages available for the production of ship lines, which include tools for the fairing and smoothing of the hull shape, and calculation of immersed volumes, areas, and initial stability.

It is reasonable to assume that most hull forms are designed using computer aided design (CAD) software. This software generally allows the user to output a file containing a numerical representation of the surface of the hull. It is important that the algorithm developed here can be used independently of any particular surface definition software. Here it is assumed that the CAD software defines the surface in a right-handed global coordinate system. If this is not the case for a particular software package, appropriate transformations must be applied to the data.

It is advantageous computationally if the data representing the surface are output in the form of a topologically rectangular grid (i.e. that there are the same number of $x$ data points at each $y$ coordinate and vice versa). If the data are not output in this form, a number of methods can be implemented to create the topologically rectangular grid including spline fitting or a manual method.

\subsection{Planking methodology}

Figure 1 shows a plank located on a threedimensional body and this general case is used to demonstrate the method used within the algorithm. The upper edge of the plank follows the direction of the longitudinal lines and intersects the transverse lines. The procedure finds the position of the lower plank edge on each of the transverse lines.

In order to simplify the problem of calculating the position of the next plank edge, a local coordinate system $(\xi, \eta, \zeta)$ is constructed at the intersection point $I_{j}$. It consists of a vector $V_{L}$, defining the edge of and being tangential to the plank at the intersection point $\mathrm{I}_{j}$, and defines the $\xi$ axis of the local coordinate system. The vector $\boldsymbol{V}_{\mathrm{t}}$ is in the plane of the plank, running transversely orthogonal to its edge $\left(\boldsymbol{V}_{\mathrm{L}}\right)$ and defines the $\eta$ axis of the local coordinate system. Finally, $\boldsymbol{V}_{\mathrm{n}}$ is normal to the plank surface and is orthogonal to both $V_{\mathrm{L}}$ and $V_{\mathrm{t}}$ and defines the $\zeta$ axis of the local coordinate system.

The vector $V_{\mathrm{L}}$ is constructed at the intersection point $\mathrm{I}_{j}$ (Fig. 2) using points defining the upper plank edge or those of the data point net. Various methods

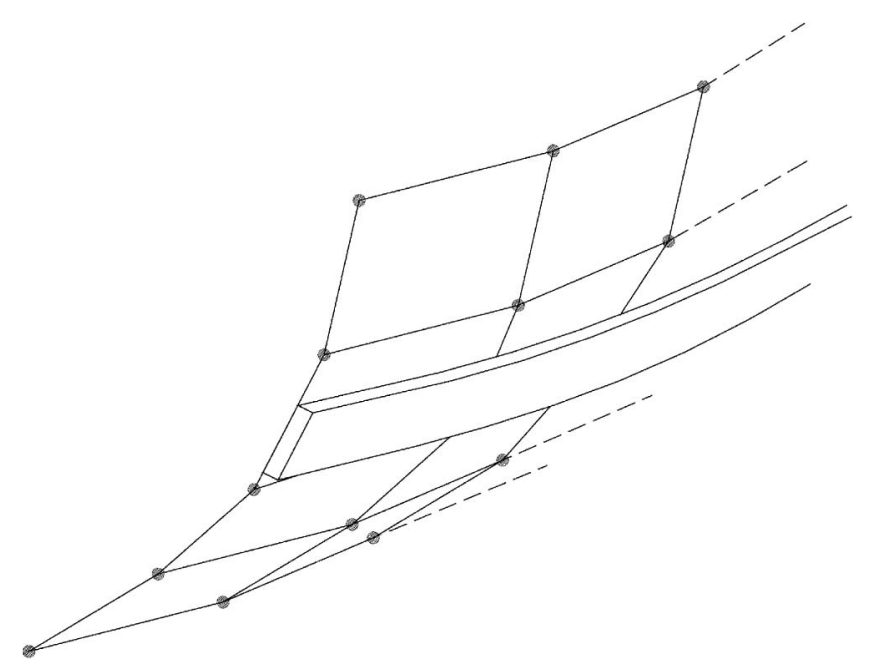

Fig. 1 Plank located on a three-dimensional surface 


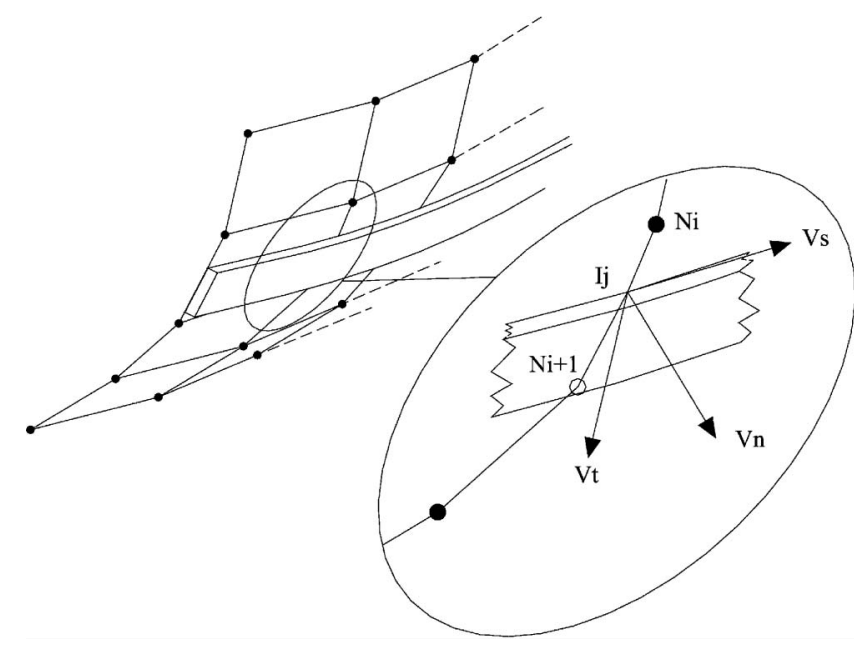

Fig. 2 Local coordinate system located at the intersection point $\mathrm{I}_{j}$

of constructing the vector $\boldsymbol{V}_{\mathrm{L}}$ can be used including linear interpolation or spline fitting. The advantages and disadvantages of each and their influence on the final outcome of the algorithm will be discussed and demonstrated later.

From Fig. 2 the next plank edge will lie on the line of the net points running girthwise from point $\mathrm{I}_{j}$. A vector $V_{\text {c }}$ can be constructed using the net points $\mathrm{N}_{i}$ and $\mathrm{N}_{i+1} \cdot \boldsymbol{V}_{\mathrm{c}}$ defines the (as yet unknown) direction along the rectangular net to the next plank edge. It is assumed that both $V_{\mathrm{L}}$ and $\boldsymbol{V}_{\mathrm{c}}$ are in the plane of the plank. Using this assumption, the vector $V_{\mathrm{n}}$ normal to both $V_{\mathrm{L}}$ and $\boldsymbol{V}_{\mathrm{c}}$ can be constructed. The final vector $\boldsymbol{V}_{\mathrm{t}}$, normal to both $\boldsymbol{V}_{\mathrm{L}}$ and $\boldsymbol{V}_{\mathrm{n}}$ can be constructed. Using the known vectors $\boldsymbol{V}_{\mathrm{L}}$ and $\boldsymbol{V}_{\mathrm{c}}$, calculated from the net point data, the remaining vectors are calculated using the relationships

$$
\begin{aligned}
& V_{\mathrm{n}}=V_{\mathrm{c}} \times V_{\mathrm{L}} \\
& V_{\mathrm{t}}=V_{\mathrm{L}} \times V_{\mathrm{n}}
\end{aligned}
$$

As discussed, $\boldsymbol{V}_{\mathrm{L}}$ and $\boldsymbol{V}_{\mathrm{c}}$ are coplanar in the plane of the plank. To find the distance to the next plank edge (the projected plank width) in the direction of $V_{c}$, the angle between $V_{c}$ and $V_{t}$ must be found. It is also important to identify the position of $\boldsymbol{V}_{\mathrm{t}}$ with relation to $V_{\mathrm{c}}$ as it will influence the sign of the $\xi$ coordinate. With the origin of the local coordinate system $(\boldsymbol{\xi}, \boldsymbol{\eta}, \boldsymbol{\zeta})$ located at the intersection point $\mathrm{I}_{j}$, the position of the next plank edge can be defined by the relationship

$$
(\xi, \eta, \zeta)=( \pm w, 0, w \tan \theta)
$$

where $w$ is the manufactured plank width and $\theta$ is the angle between $\boldsymbol{V}_{\mathrm{t}}$ and $\boldsymbol{V}_{\mathrm{c}}$. Two specific scenarios can occur at this point; where the next plank edge lies on the present girthwise segment and where it lies on any of the subsequent girthwise segments. The methods used to deal with these two scenarios have been described in detail in reference[4].

The entire procedure is repeated for each girthwise section until all the sections have been filled with planks. The surface of the body has now been planked and the curvature analysis can begin. However, there are a number of points that must be considered prior to beginning the planking procedure; these include planking direction and the starting line of data.

\subsection{Choice of planking direction and starting line}

The choice of planking direction will have a significant influence on the stress induced on the plank due to forming. In the positioning of planks on to a surface, the force required to ensure a close fit between adjacent planks and the stresses that the curvature of the plank causes are important parameters.

The easiest and most commonly found method of planking a hull form is longitudinally. The technique involves laying planks longitudinally on to a network of frames. Each plank is forced to lie against its neighbour to create a watertight outer skin. Planking a vessel transversely could also be considered and may have certain advantages. However, it also has some major disadvantages. A number of hull forms were examined to assess a transverse planking strategy. It was found that the relatively tight bilge radius, often found on high-speed vessels, prevented a successful transverse planking strategy. The stress caused by the orthogonal bending of the plank far exceeded the allowable stress of the plank used in the investigation. Diagonal planking is also used in the construction of wooden yachts and boats. However, this method involves tapering each plank to fit seamlessly against its neighbour. As prismatic planks are being dealt with, tapering of the planks is not considered an option, as it would be both expensive and labour intensive. In the present study, only planking from keel to sheer and from sheer to keel are examined.

Depending on the planking strategy being adopted, the planking procedure requires an appropriate starting line of data points which will define the first plank edge. Based on the longitudinal planking directions mentioned above, the starting lines of data could be located at the hull discontinuities such as the keel line, sheer line, or chine line. As an example, a sketch of a hull form is shown in Fig. 3 and can be used to demonstrate the effect of choosing the starting line of data. In Fig. 3(a) the hull is planked from keel line to sheer line. Here the starting line of data 


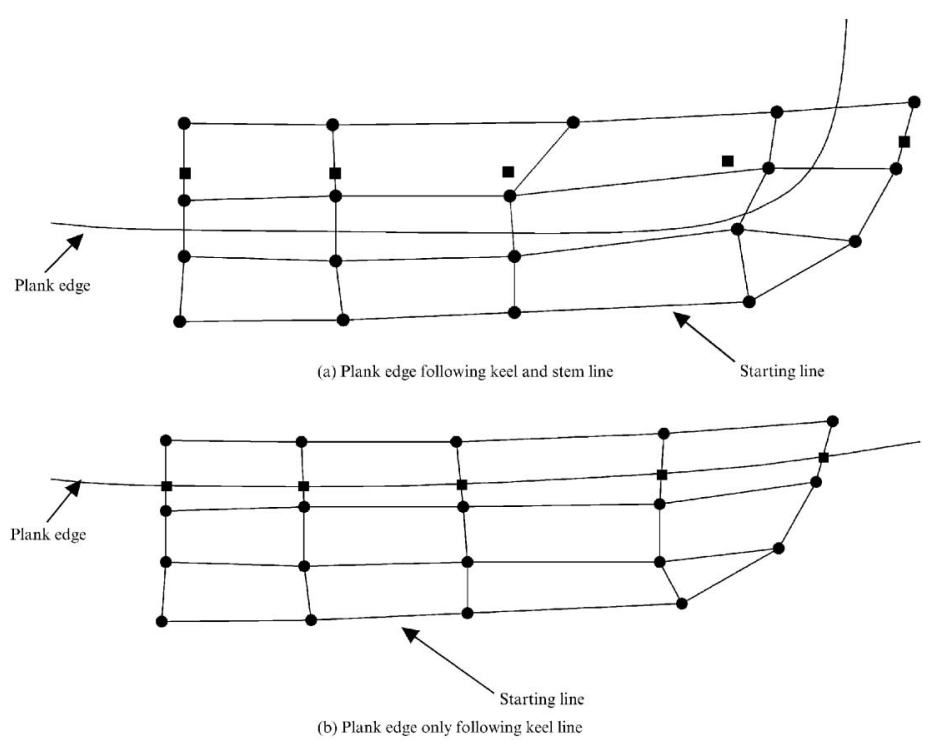

Fig. 3 Effect of different planking strategies

lies along the keel line and up the stem line. It is clear to see that any planks placed along this line will run along the keel and sweep up the stem and off the hull through the sheer line. If, however, the starting point were located on the keel line alone, then the plank would sweep off through the stem line (Fig. 3(b)). The former option would produce a plank position with high curvature and therefore high stress, whereas the latter option would produce less curvature or induced stress.

\subsection{Output from planking algorithm}

The output from the planking algorithm consists of the plank positions on the three-dimensional body. The positions can then be used to determine the effect of the choice of planking direction or the effect of the hull characteristics. This is carried out by calculating the curvature of the planks combined with knowledge of the plank geometry to produce a stress induced by forming.

With respect to hull-form geometry a number of questions can be addressed.

1. What is the maximum curvature (minimum radius of curvature) that each plank exhibits as it lies on the surface?

2. What percentage of the hull can be planked if the curvature is not allowed to exceed a certain value?

3. How can the design be modified to reduce the curvature of the planks?

The last question leads to another important factor with respect to high-speed hull forms.
4. What effect will modifications designed to improve suitability for planked construction have on hull performance?

In addressing the first three questions the plank curvatures must be known. It is important at this stage to ensure that the correct curvatures are being analysed and truly represent the curvatures that are developed within the plank.

\subsection{Curvature}

One fundamental concern in the design of a hull form is the determination of fairness or smoothness of the surface. In general, one of the most commonly used metrics in ship design software is Gaussian curvature. Gaussian curvature is the product of the minimum and maximum principal curvatures located at each and every point on a surface. However, in the context of the current study, the curvatures of interest are those in the plane of and orthogonal to the plank surface and are therefore related to the plank rather than the underlying surface. These cannot be easily obtained from knowledge of the magnitude of the Gaussian curvature.

At any point on a three-dimensional curve a moving trihedron can be constructed. This consists of three orthogonal vectors and three orthogonal planes. The orthogonal vectors are those constructed as the local coordinate system in section 2.2. Each of the three planes contains the point on the curve and one of the vectors. The planes are known as the osculating plane (containing $V_{\mathrm{L}}$ ), the rectifying plane 
(containing $\boldsymbol{V}_{\mathrm{c}}$ ), and the normal plane (containing $\boldsymbol{V}_{\mathrm{n}}$ ). Figure 4 shows the construction of a moving trihedron at a point $\mathrm{P}$.

The normal vector in Fig. 4 points in the direction that the curve is turning and towards the centre of curvature. The magnitude of the normal vector will provide the magnitude of the radius of curvature at the point $P$. In a global coordinate system this normal vector will provide the principle curvature. In this case, interest lies in the curvature in the planes of the plank and so the normal vector is found in the local coordinate system. The principal normal vector found using any coordinate system could be divided into its coordinate components. An equation for each component in the principal curvature allows the curvature orthogonal to and in the plane of the plank to be found.

The radius $R$ of curvature for any point on a space curve can be given by

$$
\frac{1}{R}=\frac{\left|P^{u} \times P^{u u}\right|}{\left|P^{u}\right|^{3}}
$$

The superscript $u$ represents differentiation with respect to the parametric variable $u$.

By dividing up the principal normal vector into its local coordinate component parts the expressions for orthogonal and in-plane curvatures respectively are obtained as

$$
\begin{aligned}
\frac{1}{R(\eta)} & =\frac{\mathrm{d}^{2} \eta / \mathrm{d} \xi^{2}}{\left[1+(\mathrm{d} \eta / \mathrm{d} \xi)^{2}\right]^{3 / 2}} \\
\frac{1}{R(\zeta)} & =\frac{\mathrm{d}^{2} \xi / \mathrm{d} \xi^{2}}{\left[1+(\mathrm{d} \xi / \mathrm{d} \xi)^{2}\right]^{3 / 2}}
\end{aligned}
$$

The calculation of the derivatives can be undertaken either numerically or by using a curve- or spline-fitting routine.

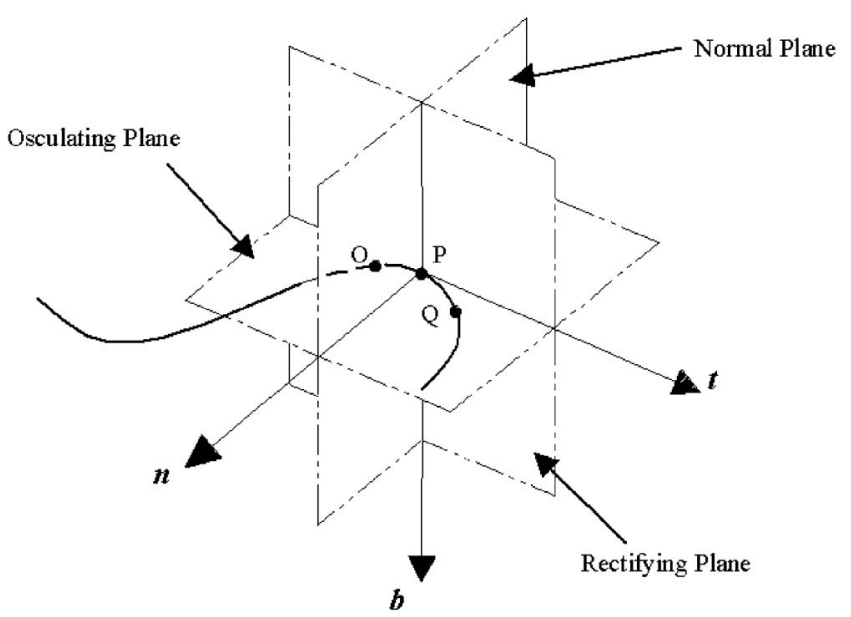

Fig. 4 Construction of a moving trihedron at point $\mathrm{P}$

\subsection{Derivative calculation}

In the calculation of the derivatives for the local coordinate system in section 2.2, two techniques were described: a numerical method and a spline- or curve-fitting method. Initially the numerical method was used. Here central differencing was employed as it is relatively simple and extremely robust. However, when a similar method was used for the curvature derivatives in section 3.1, the results became very erratic. This phenomenon only occurred when large numbers of points were used to define the curve. This was surprising as it was felt that a piecewise linear method would become more accurate with increasing curve definition.

The problem was demonstrated by planking a cylinder around its circumference; the cylinder had a radius of curvature of $6 \mathrm{~m}$ (equivalent to a curvature value of $1.667 \times 10^{-1} \mathrm{~m}^{-1}$ ). In this case, the radius of the cylinder predicts the orthogonal curvature of the plank. Using the linear interpolation method with nine points defining the cylinder the curvature was found to be relatively smooth and moderately accurate. 21 points increased the accuracy of the calculation. However, when 151 points were used, then the curvature developed an oscillation about the actual radius of the cylinder. The comparison between 9,21 , and 151 defining points is shown in Fig. 5.

The problem arises in the definition of the local coordinate system. Although the raw data can be assumed to be extremely accurate for the cylinder, the transformation of the data into the local coordinate system induces an error in the data. This is

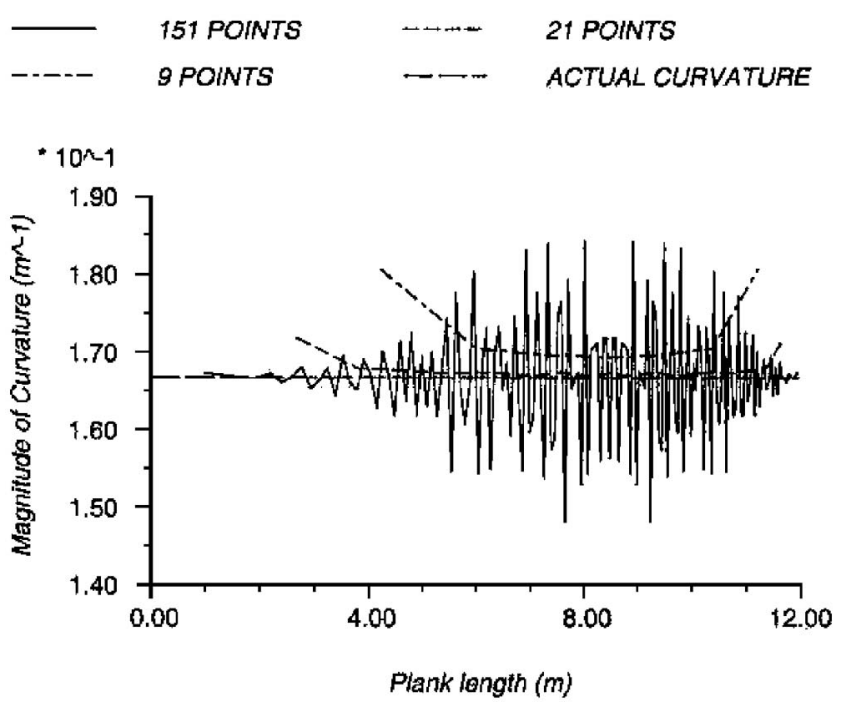

Fig. 5 Comparison of the curvature result for a cylinder defined using nine, 21, and 151 data points 
because linear interpolation is used to calculate the local coordinate system. This results in flat and even concave points on the cylinder surface. Therefore, the curvature solutions for curves with high definition must be addressed. However, it is common in naval architecture to define hulls with 21 or 41 stations and waterlines. If this convention is followed then induced numerical error can be kept to a minimum.

The use of a spline or curve to represent the plank is one solution to eliminate the numerical inaccuracies of using linear interpolation to calculate curvature. However, a certain amount of control of the surface shape may be lost. If it is assumed that the underlying data are accurate, an inappropriate spline-fitting procedure may distort the surface and lead to unrepresentative curvatures. A cubic spline was used to represent the cylinder surface and a least-squares method was used to assess the level of fit of the cubic spline. The resulting derivatives are then calculated and the results showed an increase in accuracy compared with the 151-point linear interpolation method previously described. This method is much more time consuming to implement in the context of a practical ship form, and the large number of spline combinations that are assessed for best fit could make automatic implementation difficult. For this reason it was decided to produce results using the linear interpolation method with a suitable density of points to produce reliable results without curvature oscillations.

\section{APPLICATION OF PLANKING TOOL TO EXISTING HULL FORMS}

A number of well-documented existing hull forms have been modelled and subsequently planked using an implementation of the planking algorithm described in the previous section. By examining wellknown hull forms representative of modern highspeed vessels the hull parameters that influence the success of a planked construction technique can be identified. Based on the results obtained from this study conceptual designs for high-speed craft suitable for planked construction can be developed. In all cases the hulls are planked either from keel to sheer or from sheer to keel.

The hull forms examined are from the NPL roundbilge displacement series [5], the extended NPL series [6-8], and the Series 64 methodical series [9]. It can be said that the largest length-to-breadth ratio hulls in the extended NPL series [7] are reasonably representative of present high-speed catamaran forms.
The planking algorithm was applied to three hull forms, namely NPL Parent [5], NPL 6a [7], and Series $645 \mathrm{~s}$ [10], all scaled to $50 \mathrm{~m}$ overall length. These are shown in Fig. 6. Each hull was planked using a plank width of $0.6 \mathrm{~m}$. The plank width was based on the

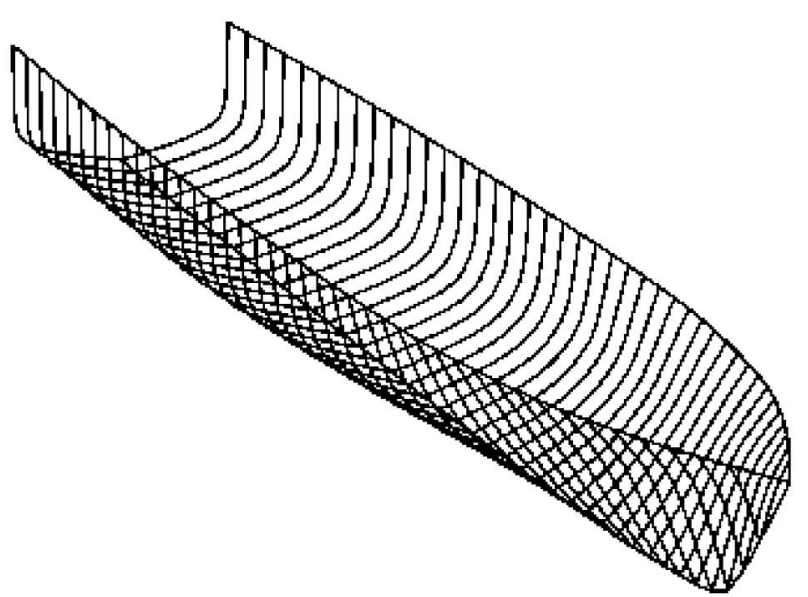

(a) NPL parent hull-form
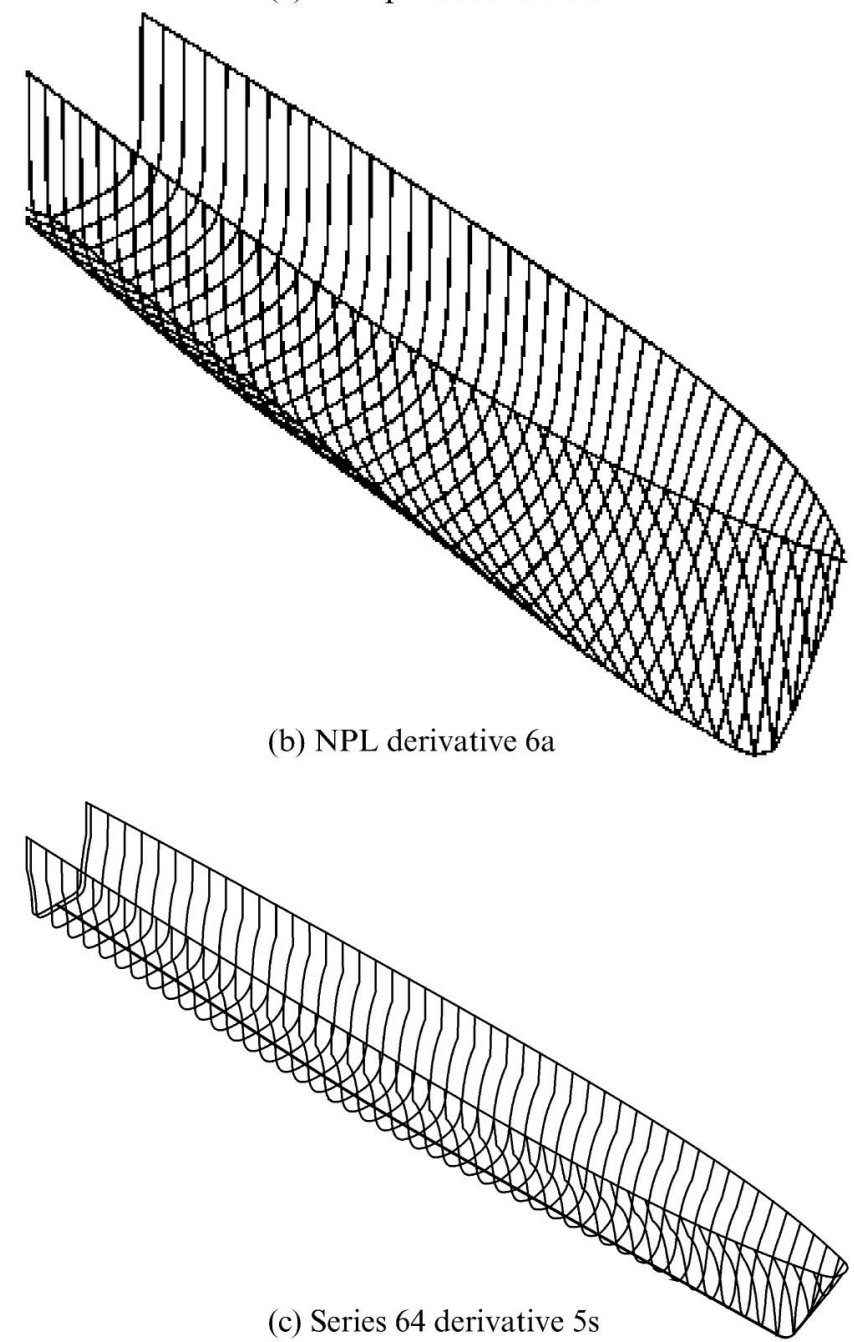

Fig. 6 Existing hull forms examined 
geometry of a commercially available pultruded glass-reinforced plastic plank. The properties of the plank were used to assess the stress induced on the plank through forming. The plank is known as the advanced composite construction system (ACCS) and is shown in Fig. 7. Details of the plank's geometry and material properties are presented in Table 1.

In the current study, the relationship between curvature and stress is approximated in accordance with linear bending theory by

$$
K_{\max }=\frac{1}{R_{\min }}=\frac{\sigma_{\max }}{E_{x} y_{\max }}
$$

where $K$ is the curvature, $R$ is the radius of curvature, $y$ is the distance from the neutral axis, $E_{x}$ is the longitudinal Young's modulus of the plank, and $\sigma_{\max }$ is the maximum allowable forming stress.

When deciding on the allowable forming stress, careful consideration must be given to the material behaviour. Some FRP composite materials, such as those consisting of glass and polyester, are prone to microcracking at strains well below the failure strain. The value of the strain at which this microcracking occurs depends on the exact nature of the material; in the current study a relatively conservative assumption is made that the maximum working stress is

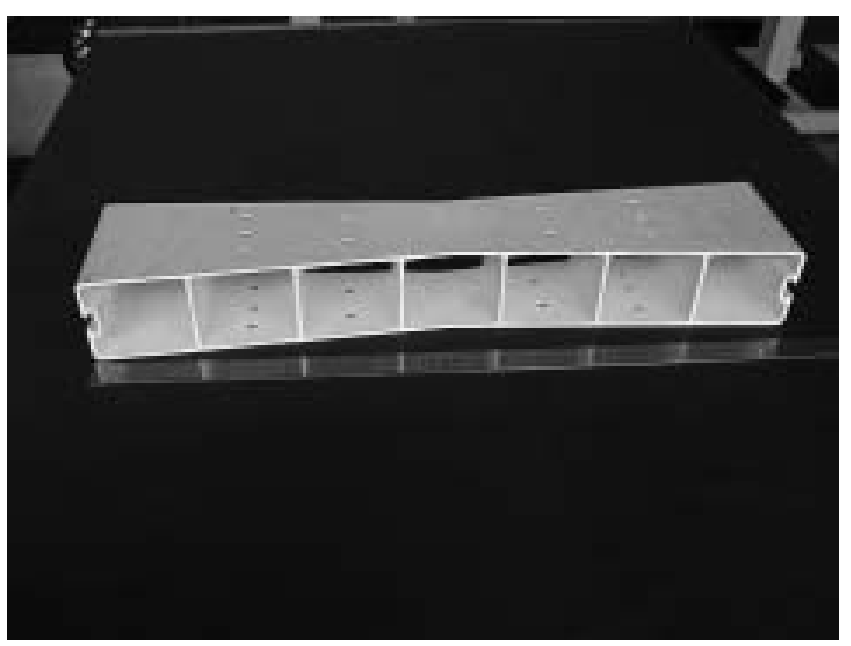

Fig. 7 ACCS pultruded GRP plank

Table 1 ACCS plank geometric and material properties

\begin{tabular}{llll}
\hline Description & Symbol & Value & Units \\
\hline Young's modulus & $E_{x}$ & 14.5 & $\mathrm{GPa}$ \\
Breadth of plank & $B$ & 600 & $\mathrm{~mm}$ \\
Depth of plank & $D$ & 80 & $\mathrm{~mm}$ \\
Longitudinal tensile strength & $\sigma_{x}$ & 485 & $\mathrm{MPa}$ \\
\hline
\end{tabular}

limited to 10 per cent of the ultimate tensile strength; for the ACCS plank discussed here, the maximum working stress is assumed to be $48.5 \mathrm{MPa}$.

Of course the proportion of strength available for forming must only be a small proportion of the allowable working stress. In the context of this example the allowable forming stress is limited to 10 per cent of the allowable working stress (equivalent to 1 per cent of the ultimate tensile strength of the panel) and is assumed to be $4.85 \mathrm{MPa}$. If the plank is subjected to orthogonal curvature only, then, based on the plank dimensions given in Table 1, the maximum allowable curvature is $8.362 \times 10^{-3} \mathrm{~m}^{-1}$. A similar calculation for a plank subjected to in-plane curvature only gives a maximum allowable curvature of $1.115 \times 10^{-3} \mathrm{~m}^{-1}$. In practice, for many hull forms, the plank will be curved in both directions simultaneously; however, an initial indication of the feasibility of using a planking technique may be obtained by considering these curvatures independently.

The results presented in Fig. 8 show the in-plane and orthogonal curvatures and the rate of change in twist angle for the first plank on the three test hulls planked from keel to sheer. Figure 9 shows the curvatures and twist results for the first plank when the hull forms are planked from sheer to keel. The results indicate that the orthogonal curvature induced on the planks is within the maximum allowable value. However, the in-plane curvature even for the first plank exceeds the limiting value, indicating that the forming stress resulting from in-plane curvature is an undesirably high proportion of the working strength of the plank. This forming stress must be reduced if planking methods are to be successful.

The development of in-plane curvature is not a direct consequence of any individual hull parameter. It can reasonably be assumed that, when planking from keel to sheer, some in-plane curvature may be developed owing to the curvature of the keel line; likewise, when planking from sheer to keel, the sheer line may not be straight. However, in both cases, the curvature of the starting line does not account for the large amounts of in-plane curvature found in the results.

It can be shown that in-plane curvature is developed by the combination of plank twist and orthogonal curvature. For a single plank, it is possible to choose a path over a non-prismatic hull surface which minimizes the energy related to forming stresses in some average sense. If the plank is wide compared with its thickness, then the bending stiffness in plane will be greater than the bending stiffness out of plane. It could therefore be 


\section{NPL Parent \\ NPL $6 a$ \\ -.-.- Sories 645 s}

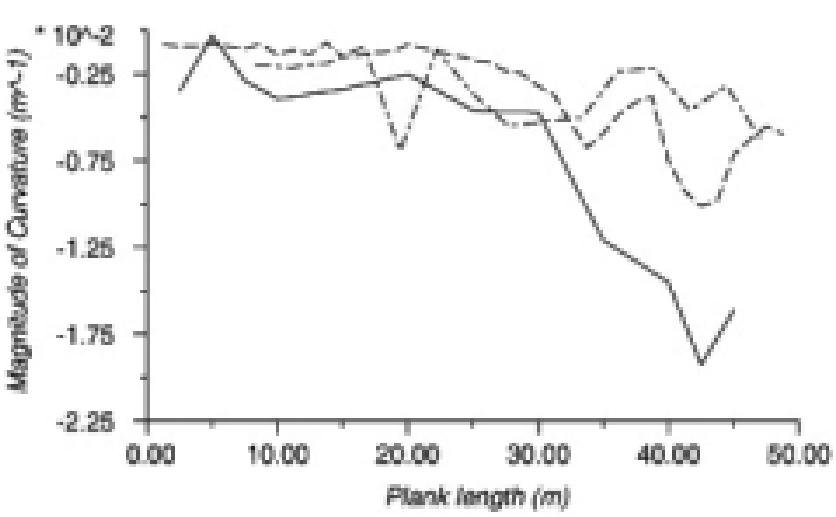

(a) Orthogonal curvature

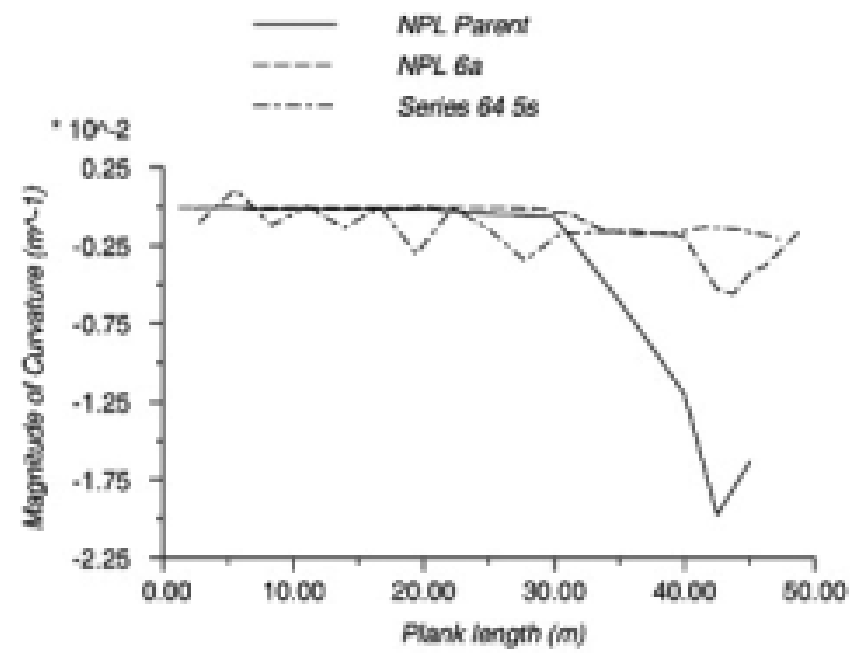

(b) In-plane curvature

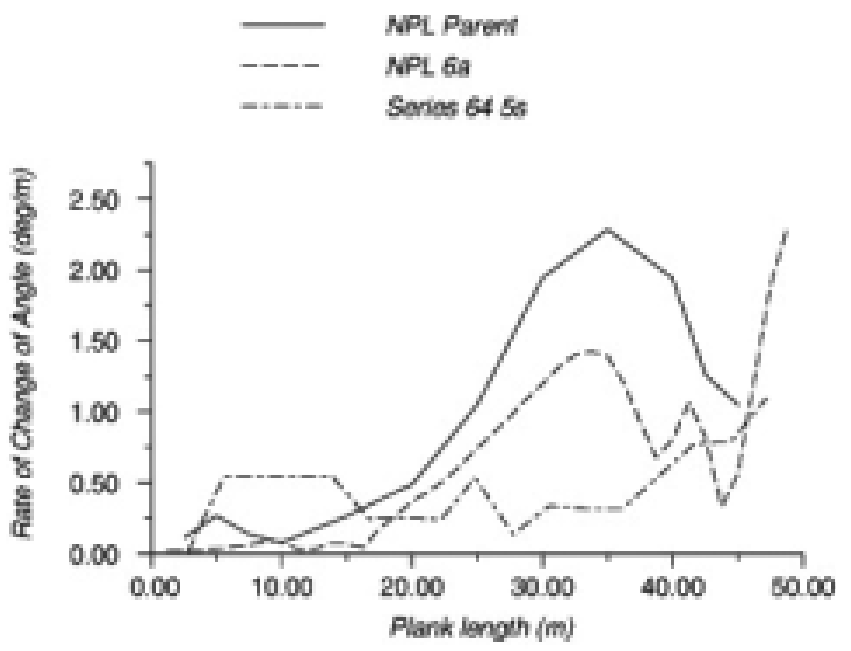

(c) Twist

Fig. 8 Curvature and twist of first planks on hull forms planked from keel to sheer
NPL Parent

NPL 68

Series $645 s$

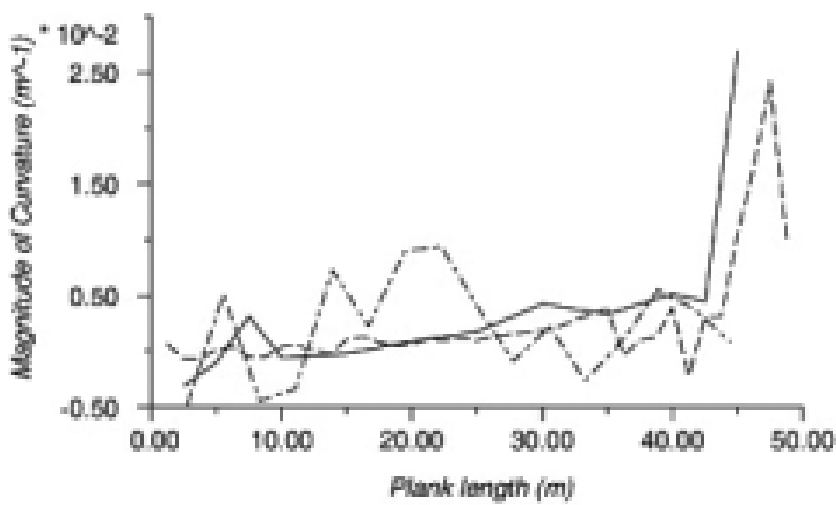

(a) Orthogonal curvature

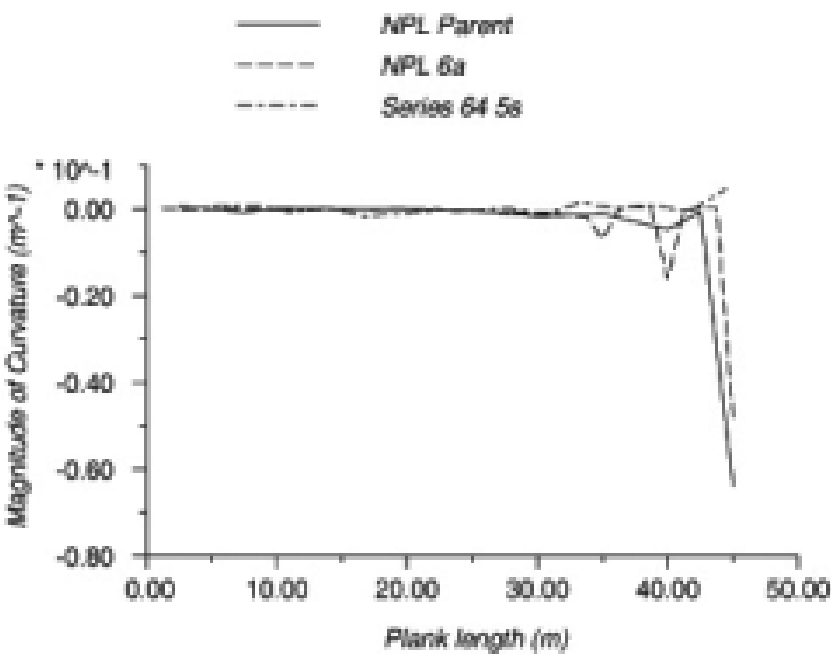

(b) In-plane curvature

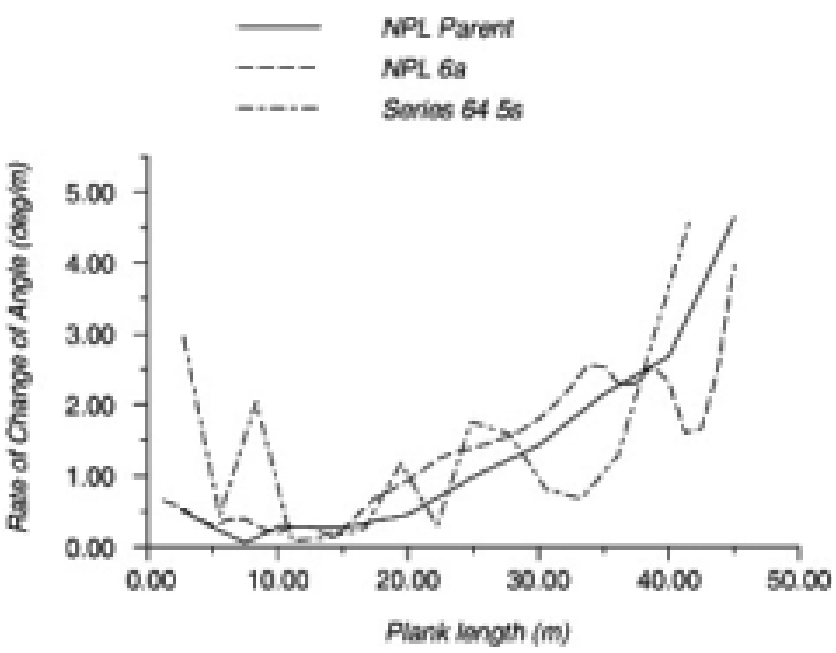

(c) Twist

Fig. 9 Curvature and twist for first planks on hull forms planked from sheer to keel 
expected that the plank would follow a path that predominantly minimizes in-plane curvature. However, if the same approach is applied to choose the path of a second plank, the two planks will not generally lie adjacent to each other everywhere, resulting in a discontinuous surface, and therefore a nonwatertight hull form. In order to generate a practical hull form, the second plank must be constrained to follow the edge of the first plank, leading to additional stresses.

In traditional boatbuilding these stresses are reduced by 'splining' the second plank to fit the curvature of the first. However, if the construction is confined to prismatic planks, the orthogonal curvature and the twist of the hull surface, combined with the requirement for a continuous watertight surface, force the development of additional in-plane curvature.

The curvatures and twist of the first plank of the NPL parent form, planked from keel to sheer (Fig. 8), can be used to demonstrate the development of in-plane curvature due to twist and orthogonal curvature.

The high in-plane curvature located at approximately $32 \mathrm{~m}$ (Fig. 8(b)) is due to the combination of twist and orthogonal curvature as the plank crosses the bilge radius. The hull geometry in the region of the first plank changes from being a relatively horizontal flat-bottomed section near the stern to a nearly vertical section near the bow, producing the twist. As the hull becomes narrower towards the bow, orthogonal curvature is introduced, the combination resulting in in-plane curvature. Conversely, the plank located at the sheer line (Fig. 9) has less twist and a slightly greater orthogonal curvature, but overall less in-plane curvature.

Based on the results obtained from the study of well-known high-speed hull forms, the amount of double curvature of these hulls results in the combination of twist and orthogonal curvature, which ultimately leads to undesirable in-plane curvature in a hull constructed with prismatic planks. In order to take advantage of the possible reduction in hull construction cost of a vessel by using a planked construction technique, the hull form must be designed with the intention of reducing double curvature as far as possible. However, since fuel costs form a major proportion of the operating costs of highspeed vessels, the potential reduction in construction cost must not be offset by increased fuel costs due to higher-power requirements, or by reduced speed. Thus the reduction in the doubly curved area must not be achieved at the expense of increased hydrodynamic resistance.

\section{CONCEPTUAL DESIGN OF A SIMPLIFIED HULL FORM SUITABLE FOR PLANKED CONSTRUCTION}

The study of existing hull forms indicates that a reduction in double curvature is a key goal in increasing the ease with which ship hulls may be constructed using prismatic planks without incurring excessive forming stresses. A concept design study was then carried out in order to investigate the feasibility of reducing doubly curved plating without increasing hydrodynamic resistance.

The concept design study falls under the heading of design for production, which in a marine sense is the design for minimum cost of ship production through ease of construction. However, this subject is far reaching and encompasses the design not only of the hull form but also of the systems required for ship operation and stretches from concept design to final out-fitting. Lamb [11] comprehensively discussed the concepts behind design for production. However, the concept design study in the present research deals only with the outer shell structure of the hull form. Lamb [11] discussed the design for production problems associated with the hull form shape and highlighted the fact that simplified forms are not new and there have been a number of studies that have investigated hull forms with straight frames from as early as 1917. In all these studies the overlying important factor is the minimization of shell plating that contains double curvature. The reason is that creating doubly curved panels requires significantly more effort than those with only single curvature, commonly referred to as developable surfaces, which can be defined as those which have one or both of the two principal curvatures equal to zero.

The above discussion on design for production for hull-form design deals exclusively with traditional plate welding construction techniques. In the case where the hull will be manufactured from preformed pultruded profiles it is already known that the planks will be developable. The aim of the present work is to redesign the hull form to reduce the double curvature present in the hull-form shape to ease construction with an alternative technique. The resulting hull form is examined hydrodynamically and compared against a suitable 'parent' form.

A round-bilged catamaran demihull form was chosen as the 'parent' form as it could be considered broadly typical of modern design practice. The NPL 6A derivative developed by Molland et al. [7] was chosen for this purpose, owing to the extensive studies that have been carried out on this series of hulls. 
A conceptual design for a highly simplified demihull form suitable for planked construction was then developed for the purposes of comparison. The hull was based on one of the forms studied by Warren and Kecsmar [12]. The form studied by Warren and Kecsmar had rectangular sections throughout its length (apart from a small bilge radius), while the beam was constant over 60 per cent of its length from the stern with a straight taper to the bow. This form is naturally singly curved above the bilge and therefore ideally suited to form the basis of an investigation into hull forms designed specifically for planked construction techniques, avoiding problems associated with double curvature.

In order to draw meaningful comparison between the parent and the simplified hull forms, a number of design parameters must remain constant between the two designs. In the current study, the length was kept constant in order to keep the Froude number constant at the design speed, while the displacement was kept constant in order to ensure that vessels had comparable functionality. Once these were fixed, the beam and draught must be chosen to maintain the displacement at the correct value. If the objective were simply to minimize the resistance, then an optimal beam-to-draught ratio could be found; however, in practice the beam of the demihulls of highspeed catamarans is often constrained by the need to accommodate appropriate machinery and propulsion. It was thus decided for the concept study to maintain the beam, in order to allow accommodation of similar sized engines and propulsion machinery. The immersed transom area was also kept constant, as it is believed to be an important parameter in the resistance of vessels with transom sterns.

Adopting these constraints, and using the WarrenKecsmar form as a starting point, a conceptual design for the highly simplified catamaran demihull form was developed which could be compared directly with the NPL 6A design. A wire frame model of the design is shown in Fig. 10. One key feature of the design from the point of view of construction is the definition of a well-defined region above the bilge radius within which a planked construction might be used.

This demihull form was planked using the planking algorithm. The results showed that there is no in-plane curvature and no twist in any of the planks located above the bilge radius. The side shell above the bilge radius is the most obvious place to apply a planked construction technique as it has been shown the planks crossing the bilge radius result in excessive undesirable in-plane curvature. The largest

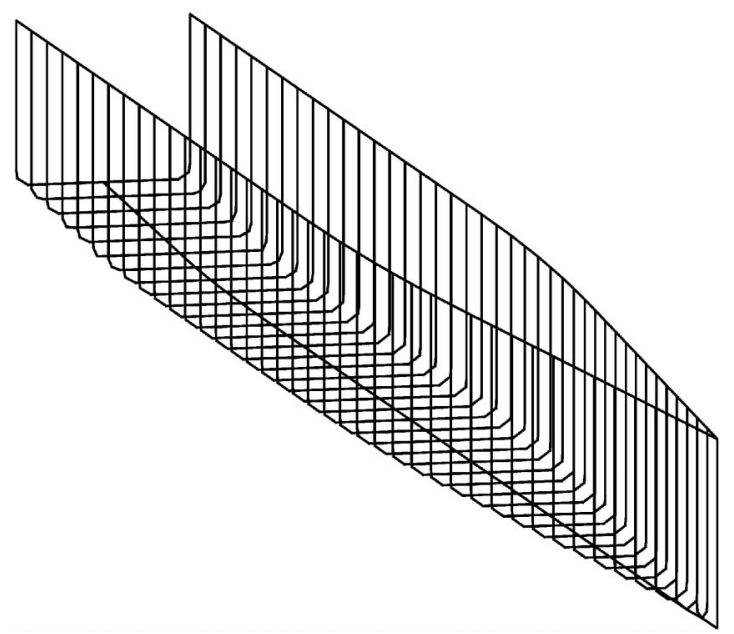

Fig. 10 Simplified hull form developed for comparative study

orthogonal curvature is located at the transition between parallel body and the taper to the bow. Figure 1la shows the comparison between the orthogonal curvature in the third plank of the two designs. The figure shows that the maximal value of the orthogonal curvature of the simplified form is slightly larger than that of the parent form and marginally exceeds the proposed maximal value based on the considerations of the forming stress indicated in section 3. In practice, it is highly likely that subtle modifications to this region would reduce the orthogonal curvature produced to a value within the limit proposed.

The main advantage of the simplified form results from the reduced in-plane curvature and twist compared with the parent form. Examples are shown for the third plank on each of the two designs in Figs 11 (b) and (c). As expected, the highly simplified form demonstrates zero in-plane curvature and twist, thus satisfying the proposed constraint on forming stress; the NPL 6A form displays substantial curvature and twist near the bow and violates the limit on forming stress.

Having demonstrated the improved suitability for planked construction of the simplified form compared with the parent hull, a series of model tests were carried out in order to compare the calm-water resistance of the two catamaran forms, The first adopted the NPL $6 \mathrm{~A}$ form as demihulls, while the second used the simplified form. These have been described in detail in reference [13]. Figure 12 shows the resistance 'penalty' plotted against Froude number for the highly simplified form, expressed as a percentage of the resistance of the parent form. The region of most interest for typical high-speed craft is typically $0.6<F n<0.8$. In this region the 


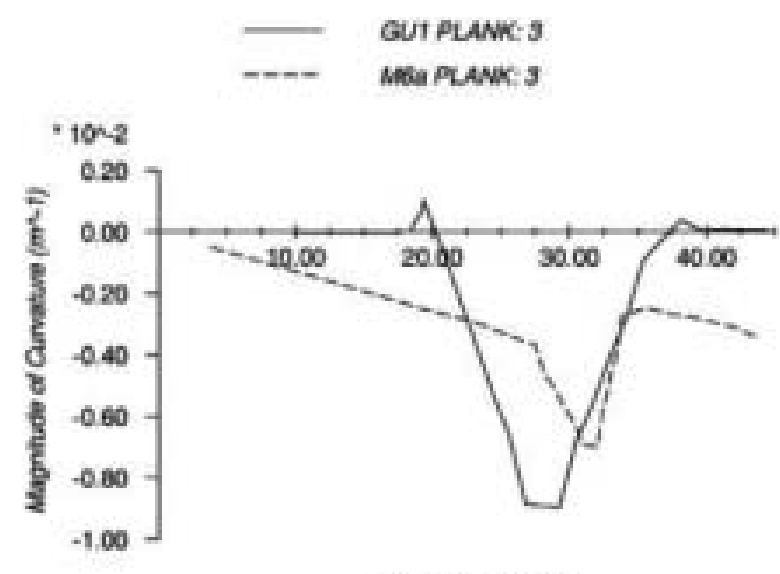

(a) Orthogonal curvature

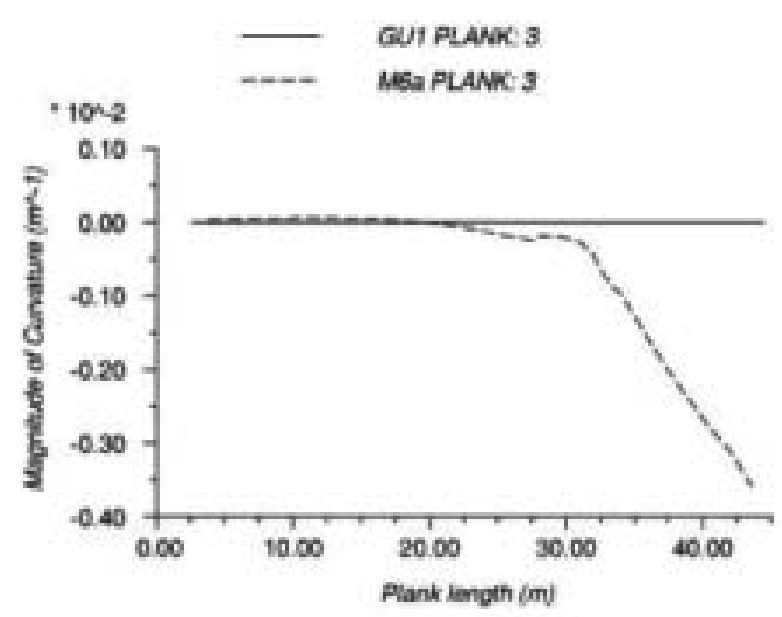

(b) In-plane curvature

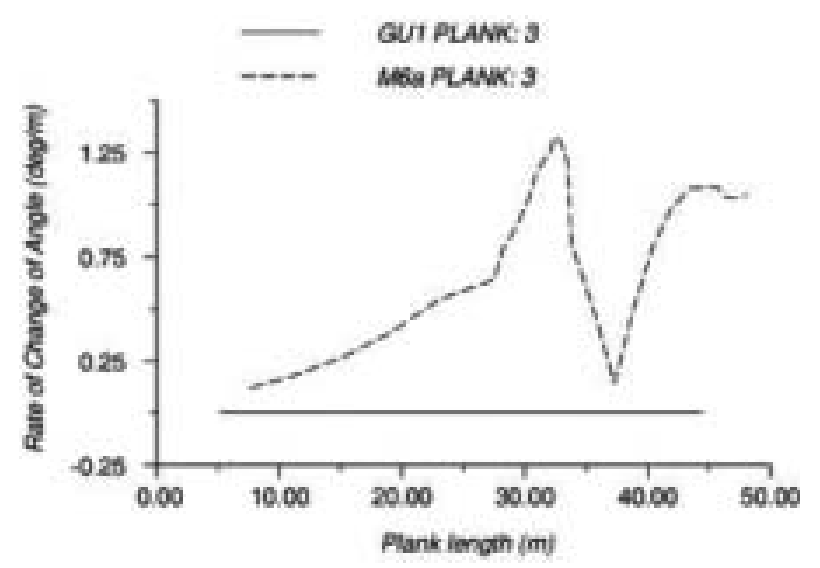

(c) Twist

Fig. 11 Curvature and twist of the third plank on a simplified hull form compared with the parent form

resistance penalty for the simplified form is between 0 and 3 per cent; however, it must be remembered that the simplified hull was developed without any attempt to optimize the geometry for resistance.

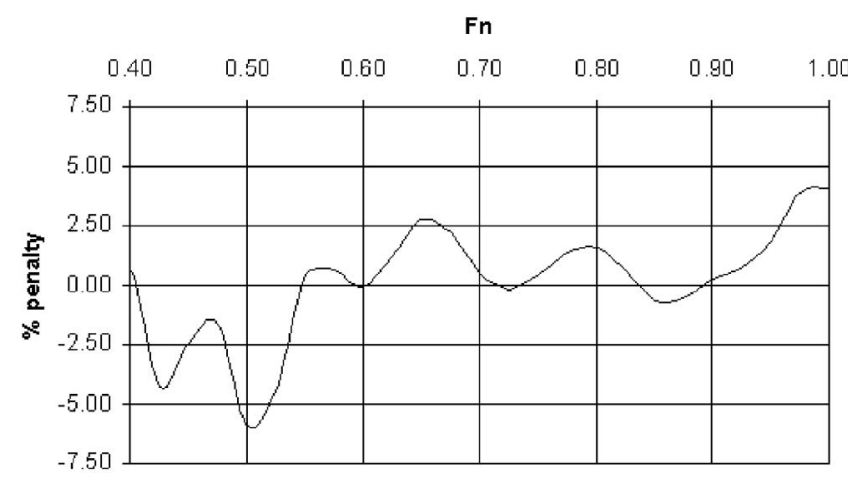

Fig. 12 Resistance penalty for a simplified hull (compared with the parent hull)

\section{DISCUSSION}

Many issues remain to be resolved before the construction of ships using pultruded FRP planks could be considered in practice. In particular, the transverse strength of the material is a potentially difficult issue. Joining the planks along edges (seams) may not be a major problem, as existing systems have proved quite successful; however, butt joints may be more problematic. This is of particular concern in the construction of ships long enough that modular construction is desirable, and where limitations related to road transport restrict the lengths of planks which might be delivered. In addition, since it is clear from the current study that some regions of the ship would have to be constructed using more conventional techniques, a system for joining pultruded planks on to conventional mouldings and, indeed, other materials would be required.

Nonetheless the study has shown that it is potentially feasible to design a high-speed catamaran demihull so that a substantial proportion may be constructed using an FRP planking system without significantly compromising hydrodynamic resistance. While the focus of the current study was placed on the construction of planked ships, it should be noted that there might also be substantial benefits to be gained in reducing the geometric complexity using traditional plating construction techniques.

\section{CONCLUSIONS}

The planking algorithm has been shown to provide a useful tool for the evaluation of present-day and future hull form designs for construction using a planked technique. It has been shown that presentday forms such as the NPL form are unsuitable for 
construction using planking technologies owing to high proportions of the surface containing double curvature.

Simplified forms provide an ideal opportunity to exploit the advantages of planked construction technologies by dramatically increasing the ratio of single to double curvature, without significantly affecting the hydrodynamic resistance. However, it has been shown that the planking of regions of the hull near or at the bilge radius still introduce large levels of undesirable in-plane curvature. Therefore, it is suggested that only regions of the hull that are located above the bilge radius would be constructed using a planked technique.

A conceptual design of a simplified form has been developed so that a large proportion of the hull surface could be constructed using a planking technique, without significantly compromising hydrodynamics resistance. The simplified form could also offer significant benefits in conventional plated construction.

\section{ACKNOWLEDGEMENTS}

This work was supported by the UK Engineering and Physical Sciences Research Council under Grant GR/M55084 'Use of pre-formed composites for costeffective fast marine craft construction'.

The authors would like to thank Professor Tony Molland of the University of Southampton for the loan of the NPL 6A model.

\section{REFERENCES}

1 Phillips, S. The potential for pre-formed composites in the high speed craft market - preliminary report. Report STL/220/01, Seaspeed Technology Limited, Chichester, West Sussex, 2000.

2 Cadei, J. and Stratford, T. The design, construction and in-service performanceof the all-composite Aberfeldy footbridge. Advanced Polymer Composites for Structural Applications in Construction, 2002, pp. 445-455 (Thomas Telford, Southampton).

3 Mottram, T. Why is the design of primary connections a major engineering issue? 6th CoSACNet Workshop, Warwick, UK, 2002.

4 Boyd, S. W. The use of pre-formed composites for fast marine craft construction. MSc thesis, University of Glasgow, Glasgow, 2002.

5 Bailey, D. The NPL High-speed Round Bilge Displacement Hull Series, 1976 (Royal Institution of Naval Architects, London).

6 Insel, M. and Molland, A. F. An investigation into the resistance components of high-speed displacement catamarans. Trans. R. Instn Nav. Architects, 1991, 134, 1-11.

7 Molland, A. F., Wellicome, J. F., and Couser, P. R. Resistance experiments on a systematic series of high-speed displacement catamaran forms: Variation of length-displacement ratio and breadth-draught ratio. Trans. R. Instn Nav. Architects, 1995, 137, 55-68.

8 Molland, A. F. and Lee, A. R. An investigation into the effect of prismatic coefficient on catamaran resistance. Trans. R. Instn Nav. Architects, 1997, 139, 157-162.

9 Yeh, H. Y. H. Series 64 resistance experiments on high-speed displacement forms. Marine Technology, 1965, 2, 248-272.

10 Molland, A. F., Wellicome, J. F., Temeral, P., Cic, J., and Taunton, D. J. Experimental investigation of the seakeeping charactistics of fast displacement catamarans in head and oblique seas. Trans. R. Instn Nav. Architects, 2001, 143, 79-98.

11 Lamb, T. Design/production integration. In Ship Design and Construction (Ed. T. Lamb), 2003 (Society of Naval Architects and Marine Engineers, Jersey City, New Jersey).

12 Warren, N. and Kecsmar, J. Practical design aspects inthe hydrodynamics of fast craft. In Proceedings of the International Conference on the Hydrodynamics of High-Speed Craft, 1999 (Royal Institution of Naval Architects, London).

13 Boyd, S. W., Day, A. H., and Winkle, I. E. Design considerations for lightweight high-speed ships using planked construction. In Proceedings of the 6th International Conference on Fast Sea Transportation (FAST '01), Southampton, 2001, Vol.3, pp. 231-241. 